\title{
Uso del cine comercial como herramienta docente en estudios en ciencias de la salud. Una experiencia multidisciplinar y colectiva
}

\author{
Jorge Pérez, José Aramburu, Josep E. Baños, Fèlix Bosch, Juana Díez, Magí Farré, Meritxell Girvent,
} Mariano Sentí, Olga Valverde

Resumen. El uso de películas comerciales ha sido habitual para fomentar el aprendizaje en diversas experiencias universitarias, especialmente en estudios en ciencias de la salud. En el presente trabajo se presenta una dilatada experiencia con dicho método docente llevada a cabo con los estudios de biología en la Facultad de Ciencias de la Salud y de la Vida de la Universitat Pompeu Fabra. Desde el inicio de los estudios, se utilizaron diversas películas en todos los cursos de la carrera que sirvieron para fomentar el aprendizaje de objetivos docentes muy diversos. En concreto se ha utilizado habitualmente el cine como herramienta docente en siete asignaturas ubicadas en los cinco cursos del currículo. En todos los casos, la experiencia fue muy bien valorada y mantiene su continuidad en los nuevos grados de Medicina y de Biología Humana que se imparten en el centro.

Palabras clave. Biocinema. Biología. Cine comercial. Enseñanza de la ciencia. Estudiantes de ciencias de la salud. Métodos docentes.

\section{The use of popular movies in the teaching of health sciences students. A multidisciplinary experience}

Summary. Popular movies have been used in a wide range of university courses for different teaching purposes, especially in studies of health sciences. This paper describes an experience of using such didactic approach in a wide range of subjects in the bachelor of Biology at the Pompeu Fabra University. Since the beginning of the studies, several films were used to promote the learning of diverse educational objectives. Specifically, seven subjects located in the five courses of the curriculum have been commonly used films as a teaching tool. In all cases, the experience was highly valued and it is continued in the new degree studies of Human Biology and Medicine.

Key words. Bio cinema. Biology. Health science students. Popular movies. Science education. Teaching tools.

\section{Introducción}

El uso de películas comerciales ha sido habitual en diversos estudios universitarios como derecho [1], económicas [2], administración educativa [3], rehabilitación [4] o historia de la farmacia [5], pero el interés en el empleo de este método docente ha resultado particularmente notable en la enseñanza de la medicina [6-11], la psiquiatría [12-15] y la psicología [16-19].

A pesar de que dicha utilización no ha sido tan habitual como en las disciplinas citadas, también se conocen experiencias del uso del cine en la enseñanza de la biología. Meyer y Powers [20] sugirieron una lista de películas comerciales para tal fin. Rose $[21,22]$ y Chifré [23] han mostrado las ventajas del uso de algunas cintas en la enseñanza de la genética, la biología del desarrollo o la biología evolutiva. Tam- bién Kirby [24] ha fomentado el debate sobre la eugenesia a través de películas de ciencia ficción.

La Facultad de Ciencias de la Salud y de la Vida de la Universitat Pompeu Fabra (UPF) inició su actividad en el curso 1998-1999 con la licenciatura de Biología. Dichos estudios tenían una orientación biosanitaria y fueron impartidos con un proyecto educativo innovador. En estos momentos, la licenciatura está extinguida y actualmente se cursan los nuevos grados de Biología Humana y de Medicina.

La licenciatura constaba de cinco años que se cursaban en periodos de tres trimestres. Desde su inicio, en diversas asignaturas se ha utilizado habitualmente el cine comercial como método docente, si bien con diferentes objetivos. En el presente trabajo se presentan las diversas experiencias siguiendo un orden cronológico respecto a la ubicación en
Facultad de Ciencias de la Salud y de la Vida; Universitat Pompeu Fabra (J. Pérez, J. Aramburu, J.E. Baños, F. Bosch, J. Díez, M. Girvent, M. Sentí, O. Valverde). Facultad de Medicina; Universitat Autònoma de Barcelona (M. Farré). Barcelona, España.

Correspondencia: Dr. Jorge Pérez. Facultad de Ciencias de la Salud y de la Vida. Universitat Pompeu Fabra. Doctor Aiguader, 80. E-08003 Barcelona.

E-mail:

jordi.perez@upf.edu

Agradecimientos:

La experiencia narrada en el artículo ha sido reconocida con diversas distinciones. Los autores quieren agradecer al Consejo Social de la Universitat Pompeu Fabra el Premio a la Iniciativa Docente recibido en 2008 , y a la Generalitat de Catalunya, la Distinción Vicens Vives a la Calidad Docente en 2009.

Conflicto de intereses: No declarado.

Competing interests: None declared.

(c) 2014 FEM 
el currículo; se señalan sus objetivos concretos, las películas utilizadas y su valoración.

\section{Primer año de carrera}

\section{'Introducción a la biología'. Cine para evaluar la asignatura}

La licenciatura de Biología de la UPF comenzaba con una asignatura no contemplada en el currículo pero que servía para introducir a los estudiantes en el proyecto educativo del centro y en los campos de investigación de sus futuros profesores y para aumentar la motivación de los alumnos por sus estudios. La asignatura se denominaba 'Introducción a la biología', era impartida durante las dos primeras semanas de clase y servía para obtener tres créditos de libre configuración. La actividad estaba coordinada por el decano de la facultad y consistía en la impartición de algunas charlas sobre temas diversos de la biología, sobre el proyecto educativo del centro y sobre los recursos informáticos e informativos del centro.

El objetivo principal del uso del cine en esta asignatura era la realización de la evaluación final, el aumento de la motivación de los estudiantes por la investigación y el fomento de las relaciones interpersonales basadas en el respeto y la solidaridad.

En la última sesión de la asignatura, los alumnos visionaban la película Despertares (Tabla) y al día siguiente hacían la evaluación final de la asignatura, donde la mayoría de las preguntas se referían a los contenidos del programa y su relación con la película.

Los resultados académicos fueron muy positivos porque todos los estudiantes superaron siempre los estándares previstos. El atractivo de la película y los buenos resultados académicos favorecían la motivación por la carrera y la adaptación a los estudios y a los sistemas de evaluación.

Recientemente hemos comprobado que los resultados académicos de esta primera experiencia de evaluación, en la que la película es fundamental, está altamente correlacionada con el expediente final de los alumnos [25]. Este dato refuerza la percepción positiva que teníamos de la asignatura y de la actividad del cine en particular.

\section{Segundo año de carrera}

\section{‘Nutrición'. Cine para introducir el problema de la obesidad}

La asignatura de 'Nutrición' era una materia obliga- toria de 4,5 créditos y estaba ubicada en el tercer trimestre del segundo curso de carrera. En este caso se proyectaba la cinta Super size me (Tabla) para introducir a los estudiantes en el problema de la obesidad con el fin de que tuvieran una visión general de sus aspectos mas relevantes, como la epidemiología, los factores desencadenantes y sus enfermedades asociadas. Así mismo, la película servía para sugerir temas para un trabajo posterior muy relevante de la asignatura.

Durante la primera semana del curso los estudiantes veían la película. Posteriormente, en grupos de cinco o seis personas, debían seleccionar un tema relacionado con la obesidad para trabajarlo y exponerlo mediante un póster en una jornada científica al final del trimestre.

La experiencia siempre fue muy bien valorada por sus responsables a tenor de los comentarios de los estudiantes y del resultado de los trabajos expuestos durante la jornada científica.

\section{Tercer año de carrera}

\section{"Psicobiología". Cine para facilitar la discusión sobre trastornos psicopatológicos}

La asignatura de 'Psicobiología' era obligatoria con 4,5 créditos y se impartía en el primer trimestre del tercer curso de la carrera. El objetivo del uso del cine en esta asignatura era el fomento del aprendizaje de ciertos trastornos psicopatológicos como el autismo, la esquizofrenia y los problemas de memoria.

Durante los años que duró la experiencia se utilizaron cuatro películas: Rain man, Alguien voló sobre el nido del cuco, Una mente maravillosa y $A$ propósito de Henry (Tabla) y en cada curso se proyectaban dos de ellas. Unos días después de visionar cada película, se realizaba un seminario de discusión sobre el trastorno en cuestión a partir de un guión que se había distribuido previamente.

La valoración de la actividad fue muy positiva a partir de los resultados de las encuestas de satisfacción de los estudiantes y de la información recogida por la propia responsable al finalizar el trimestre.

\section{"Microbiología". Cine para introducir la relevancia de los aspectos sociales, económicos y científicos de una nueva enfermedad}

'Microbiología' era una asignatura troncal con 12 créditos y se cursaba en el tercer trimestre del tercer curso. Aquí se proyectaba la película En el filo de la $d u d a$ (Tabla) para plantear a los estudiantes los com- 
plejos aspectos sociales, económicos y científicos que convergen cuando aparece una nueva enfermedad, en este caso el sida. En concreto, se visionaba después de la explicación del tema del virus de inmunodeficiencia humana en clase. Al acabar la proyección había una discusión sobre la enfermedad.

El grado de satisfacción de los estudiantes y los excelentes resultados académicos en lo referente a la enfermedad tratada permitieron considerar a la experiencia como muy relevante.

\section{Cuarto año de carrera}

\section{'Psicología humana'. Cine como herramienta para realizar evaluaciones formativas}

'Psicología humana' era una asignatura que no estaba contemplada en el currículo de la carrera pero que se ofertaba de manera optativa a los estudiantes, los cuales podían conseguir cuatro créditos de libre elección. Esta materia se cursaba en el segundo trimestre del cuarto curso.

En este caso el uso del cine servía de soporte de dos evaluaciones formativas para consolidar los aprendizajes previstos. A la mitad del trimestre se proyectaba la película El milagro de Anna Sullivan, y al final, La naranja mecánica (Tabla). Un día después de cada proyección, los estudiantes realizaban una evaluación formativa sobre el temario cursado hasta el momento de la proyección relacionado con la película vista. Dos o tres días después, una vez corregidas las pruebas, se hacía una sesión de feedback donde los estudiantes recibían las calificaciones y donde se incidía en los objetivos docentes menos asumidos.

Esta experiencia fue muy bien valorada a partir de los resultados en las propias evaluaciones formativas y de las evaluaciones finales, donde éstas posiblemente resultaron muy mediatizadas por el aprendizaje conseguido con esta actividad docente.

\section{'Bioética'. Cine para realizar evaluación continuada y para fomentar la discusión}

La asignatura de 'Bioética' era obligatoria de 4,5 créditos y estaba ubicada en el tercer trimestre del cuarto año de carrera.

La utilización del cine en un primer momento fue para realizar una evaluación continuada y, posteriormente, para facilitar la discusión de temas bioéticos.

Hasta el curso 2006-2007 se proyectaban tres films: El doctor, Al cruzar el límite y Gattaca (Tabla). Uno o dos días después, los estudiantes contestaban unas preguntas sobre aspectos bioéticos relacionados con las películas visionadas. Tras estas evaluaciones se generaba un debate sobre el tema. El resultado de estas tres evaluaciones tenía contingencia sobre la nota final.

A partir del curso 2007-2008 se siguieron proyectando tres películas, pero incidiendo más en el fomento de la discusión que de la propia evaluación. Las películas proyectadas se asociaban a un tema relevante del programa: El doctor, con la relación entre profesional de la salud y usuario; El experimento Tuskegee, con la investigación con humanos, y Al cruzar el límite, con los límites de la investigación biomédica.

Antes de ver la película los estudiantes disponían de un guión sobre el tema y posteriormente a su visión, en grupos reducidos, se realizaba una discusión. Finalmente, los alumnos debían hacer un escrito de una página donde resaltaban el aspecto más relevante para ellos de la película o de la propia discusión. La evaluación de dichos escritos también tenía contingencia en la nota final.

La calidad de las discusiones y de los escritos entregados permitió a los responsables hacer una evaluación positiva de la actividad.

\section{Quinto año de carrera}

\section{Itinerarios profesionales. Cine para fomentar la reflexión y debatir sobre aspectos profesionales y sociales de los biólogos}

Durante el último año de carrera, los estudiantes tenían que escoger entre tres itinerarios profesionales con objetivos diferentes pero utilizando la misma metodología docente. Los itinerarios eran: 'Investigación biomédica, 'Laboratorio de análisis' e 'Industria farmacéutica y biotecnológica'. Los alumnos tenían un trimestre de formación teórica presencial y en los dos trimestres restantes realizaban prácticas profesionales reales en centros relacionados con los itinerarios.

Durante el trimestre de actividad presencial, todos los estudiantes, a pesar de tener formación específica en cada itinerario, realizaban una actividad común denominada 'Biocinema'. Dicha actividad tenía el objetivo de completar la formación y fomentar el debate sobre temas importantes relacionados con la profesión del biólogo y con su responsabilidad social.

Durante el trimestre se hacían tres proyecciones que eran seguidas de un debate moderado por uno 
Tabla. Resumen de las películas utilizadas (entre paréntesis, director y año) y sus objetivos docentes.

\begin{tabular}{|c|c|c|c|}
\hline 1. er. curso & Introducción a la biología & Despertares (Marshall, 1990) & Evaluación sumativa \\
\hline $2 .{ }^{\circ}$ curso & Nutrición & Super size me (Spurlock, 2004) & Introducción al tema de la obesidad \\
\hline & Microbiología & En el filo de la duda (Spottswoode, 1993) & $\begin{array}{l}\text { Introducción a una nueva } \\
\text { enfermedad infecciosa }\end{array}$ \\
\hline $4 .^{\circ}$ curso & $\begin{array}{l}\text { Bioética } \\
\text { (tres films cada curso) }\end{array}$ & $\begin{array}{l}\text { El doctor (Haines, 1991) } \\
\text { Al cruzar el límite (Apted, 1996) } \\
\text { El experimento Tuskegee (Sargent, 1997) } \\
\text { Gattaca (Niccol, 1997) }\end{array}$ & $\begin{array}{l}\text { Evaluación continuada } \\
\text { y fomento de la discusión }\end{array}$ \\
\hline $5 . .9$ curso & $\begin{array}{l}\text { Itinerarios profesionales } \\
\text { (tres films cada curso) }\end{array}$ & $\begin{array}{l}\text { Viaje alucinante (Fleischer, 1966) } \\
\text { La amenaza de Andrómeda (Wise, 1971) } \\
\text { Los niños del Brasil (Shaffner,1978) } \\
\text { Mi tío de América (Resnais, 1980) } \\
\text { El aceite de Lorenzo (Millar, 1992) } \\
\text { Frankenstein de Mary Shelley (Branagh,1994) } \\
\text { Estallido (Pedersen, 1995) } \\
\text { La enfermedad de Sachs (Deville, 1999) } \\
\text { El experimento (Hirschbiegel, 2001) } \\
\text { Osmosis Jones (Farrelly y Farrelly, 2001) } \\
\text { El jardinero fiel (Meirelles, 2005) }\end{array}$ & $\begin{array}{l}\text { Fomento de la reflexión y discusión } \\
\text { sobre temas de la profesión } \\
\text { y la responsabilidad social }\end{array}$ \\
\hline
\end{tabular}

de los tres profesores responsables de los itinerarios a partir de un guión repartido previamente. Durante la semana siguiente, los estudiantes, de forma individual, debían hacer un escrito de una o dos páginas con una valoración tanto de la película como de la discusión. La evaluación de dichos escritos tenía una repercusión sobre la nota de las dos asignaturas específicas de cada itinerario.

Desde que comenzó la experiencia en 2003, se han utilizado las películas siguientes: Viaje alucinante, La amenaza de Andrómeda, Los niños del Brasil, Mi tío de América, El aceite de Lorenzo, Frankenstein de Mary Shelley, Estallido, La enfermedad de Sachs, El experimento, Osmosis Jones y El jardinero fiel (Tabla).

La evaluación de la actividad de 'Biocinema', previamente publicada [26], ha sido en general muy positiva al considerar la satisfacción de los estu- diantes y de los profesores, la calidad de los escritos y la activa participación de los alumnos en las discusiones.

\section{Evaluación general y limitaciones}

Como hemos visto, el uso del cine como herramienta docente en los estudios de biología de la UPF ha sido amplio y variado. En la tabla se presentan las películas usadas y los objetivos docentes de las asignaturas donde predominaba el fomento del debate para facilitar el aprendizaje.

Hemos de destacar que las actividades comentadas no han sido producto de una planificación deliberada por parte de la institución, sino que se han producido por la iniciativa particular de los profesores implicados, todos ellos autores del presente 
trabajo. No obstante, el azar ha querido que los estudiantes hayan tenido actividades relacionadas con el cine durante todos sus años de carrera. También ha permitido que una iniciativa individual haya derivado en una actividad de colaboración docente entre todos los profesores implicados.

Como se ha comentado, cada experiencia narrada se valoró de forma positiva y, por tanto, la valoración general también es positiva. No obstante, el trabajo que presentamos adolece de una gran limitación. El objetivo fundamental del uso del cine en nuestra facultad ha sido el fomento del aprendizaje de los estudiantes. Sin embargo, el principal elemento de valoración positiva ha sido la satisfacción tanto de los profesores como de los estudiantes, pero no tenemos constancia fehaciente de que la actividad haya mejorado el aprendizaje. Tenemos la percepción subjetiva de que sí, pero necesitaríamos estudios controlados para comparar los resultados de aprendizaje de los estudiantes que hubieran seguido la actividad con aquellos que no la hubieran seguido.

A pesar de la limitación señalada, la actividad que hemos descrito apoya la idea de que el uso del cine en la docencia puede fomentar el aprendizaje tanto de competencias específicas como transversales en estudios universitarios, especialmente de ciencias de la salud [26-28]. Por ello, en los nuevos grados de Biología Humana y de Medicina, todos los implicados hemos mantenido las mismas actividades que hemos descrito en la licenciatura de Biología.

Pensamos que las experiencias narradas en el presente artículo pueden estimular a otros profesores de estudios de ciencias de la salud a utilizar el cine como herramienta docente.

\section{Bibliografía}

1. Böhnke M, Machura S. Young Tom Edison - Edison, the man: biopic of the dynamic entrepreneur. Public Underst Sci 2003; 12: 319-33.

2. Leet D, Houser S. Economics goes to Hollywood: using classic films and documentaries to create an undergraduate economics course. J Econ Educ 2003; 34: 326-32.

3. English FW, Steffy BE. Using film to teach leadership in educational administration. Educ Adm Q 1997; 33: 107-15.

4. Davis A. Using feature films in rehabilitation counselor education. Rehabil Educ 2000; 14: 169-80.

5. Landin M. The name of the rose/Der Name der Rose (1986).
Basic aspects of monastic medical-pharmacological knowledge in the middle ages. J Med Mov 2008; 4: 41-6.

6. Crellin JK, Briones AF. Movies in medical education. Acad Med 1995; 70: 745.

7. González-Blasco P. Literature and movies for medical students. Fam Med 2001; 33: 426-8.

8. Dobson T. Can medical students learn empathy at the movies? BMJ 2004; 329: 1363

9. Farré M, Bosch F, Roset PN, Baños JE. Putting clinical pharmacology in context: the use of popular movies. J Clin Pharmacol 2004; 44: 30-6.

10. Lee YM, Ahn DS. Medical-themed film and literature course for premedical students. Med Teach 2004; 26: 534-9.

11. Alexander M, Lenahan P, Pavlov A. Cinemeducation: a comprehensive guide to using film in medical education. Oxford: Radcliffe Publishing; 2005.

12. Miller FC. Using the movie Ordinary people to teach psychodynamic psychotherapy with adolescents. Acad Psychiatry 1999; 23: 174-9.

13. Rosenstock J. Beyond $A$ beautiful mind: film choices for teaching schizophrenia. Acad Psychiatry 2003; 27: 117-22.

14. Bhaga HA. Should cinema be used for medical student education in psychiatry? Med Educ 2005; 39: 972-3.

15. Sierles FS. Using film as the basis of an American culture course for first-year psychiatry residents. Acad Psychiatry 2005; 29: 100-4.

16. Anderson DD. Using films as tool for analysis in a psychology and law course. Teach Psychol 1992; 19: 155-8.

17. Conner DB. From Monty Python to Total Recall: a feature film activity for the cognitive psychology course. Teach Psychol 1996; 23: 33-5.

18. Paddock JR, Terranova S, Giles L. SASB goes Hollywood: teaching personality theories through movies. Teach Psychol 2001; 28: 117-21.

19. Kelley M, Calkins S. Evaluating popular portrayals of memory in film. Teach Psychol 2006; 33: 191-4.

20. Meyer TK, Powers C. Using science fiction to understand biological concepts; 1994. URL: http://www.woodrow.org/ teachers/bi/1994/science_fiction.html.

21. Rose C. How to teach biology using the movies science of cloning people, resurrecting the dead, and combining flies and humans. Public Underst Sci 2003; 12: 289-96.

22. Rose C. Biology in the movies: using the double-edged sword of popular culture to enhance public understanding of science. Evol Biol 2007; 34: 49-54.

23. Chifré EJ. Evolució, ciència i cinema. Revista del Col-legi de Biòlegs de Catalunya 2006; 7: 16-21.

24. Kirby DA. The devil in our DNA: a brief history of eugenics in science fictions films. Lit Med 2007; 26: 83-108.

25. Pérez J. Using the movie Awakenings to evaluate learning in the Biology Degree Program at the Universitat Pompeu Fabra. Ten years of experience. J Med Mov 2009; 5: 27-9.

26. Baños JE, Aramburu J, Sentí M. Biocinema: the experience of using popular movies with students of biology. J Med Mov 2005; 1: 42-6.

27. Fresnadillo MJ, Diego C, García E, García JE. Teaching methodology for the utilization of cinema in the teaching of medical microbiology and infectious diseases. J Med Mov 2005; 1: $17-23$.

28. García JE, García E. Cinema and literature in medical education. J Med Mov 2012; 8: 131-2. 